Check for updates

Cite this: Mater. Adv., 2021, 2, 2318

Received 30th January 2021, Accepted 20th February 2021

DOI: 10.1039/d1ma00086a

rsc.li/materials-advances

\title{
Optimizing the vectorial component of first hyperpolarizabilities of push-pull chromophores to boost the electro-optic activities of poled polymers over broad telecom wavelength bands $\uparrow$
}

\author{
Jie Zou, ${ }^{\text {ab }}$ Di Zhang, ${ }^{\text {ab }}$ Weilong Chen ${ }^{\text {ab }}$ and Jingdong Luo (1) *ab
}

\begin{abstract}
The use of thiophene as a conjugation unit has long been studied for the design and synthesis of thermally stable donor- $\pi$-bridge-acceptor ( $D-\pi-A$ ) chromophores for second-order nonlinear optics (NLO). Herein we report the rational molecular design of thiophene-bridge-based push-pull chromophores by alternating the sequence of $\pi$-conjugation units to boost the EO activities of poled polymers. Through facile synthesis, a thienyl group is directly attached to the 4-position of the $N, N$-dibutylaminophenyl donor to furnish a new 4-[5-(4-dibutylamino-phenyl)-thiophen-2-yl]-buta-1,3-dienyl donor-bridge component (APTBD) with strong tricyanovinyldihydrofuran (TCF) and $\mathrm{CF}_{3}$-TCF acceptors. Two site-isolators of $4^{\prime}$-methoxyphenyl (MeOPh) and $4^{\prime}$-dimethylaminophenyl (DMA) groups were orthometrically attached to the middle of the primary $\mathrm{D}-\pi-\mathrm{A}$ framework to study their role in changing the solubility and properties of chromophores. Through a systematic study of the linear and EO properties of this series of new chromophores and with the assist of DFT calculations, we find that alternating the sequence of $\pi$-conjugation units of thiophene-bridge-based chromophores can significantly affect the shape and dipole moment components of molecules, which are the key molecular parameters in determining the bulk NLO performance of this series of chromophores. We also find unexpected results of orthometric site-isolators in affecting the properties of these chromophores in terms of solubility, NLO property, and geometric isomerism. Our studies lead to remarkable performance improvement of these chromophores in their molecular hyperpolarizabilities and effective translation to large $r_{33}$ values up to $106.1 \mathrm{pm} \mathrm{V}^{-1}$ at $1304 \mathrm{~nm}$ and $88.7 \mathrm{pm} \mathrm{V}^{-1}$ at $1541 \mathrm{~nm}$ in poled polymers.
\end{abstract}

\section{Introduction}

Donor- $\pi$-bridge-acceptor ( $\mathrm{D}-\pi-\mathrm{A})$ chromophores with large nonlinear optical (NLO) responses are of intense research interest as the key-enabling molecular component for optical communications, information processing, sensing, and bioimaging applications. ${ }^{1-4}$ Over the past two decades, structure-property relationship analyses based on extensive theoretical and experimental studies have led to rational molecular design and synthesis of dipolar push-pull chromophores exhibiting very large molecular hyperpolarizabilities ( $\beta$ values), some of which have been used as the feedstock of organic and polymeric electro-optic (EO) materials for high-performance photonic devices. ${ }^{5-12}$

Although it is generally accepted that the molecular NLO properties of push-pull chromophores depend on the strength

\footnotetext{
${ }^{a}$ Shenzhen Research Institute, City University of Hong Kong, Shenzhen, P. R. China

${ }^{b}$ Department of Chemistry, City University of Hong Kong, Kowloon,

Hong Kong SAR, P. R. China. E-mail: jingdluo@city.edu.hk

$\dagger$ Electronic supplementary information (ESI) available: Experimental section, analytic spectra, and DFT calculation results. See DOI: 10.1039/d1ma00086a
}

of the donor and acceptor groups, as well as the nature of $\pi$-conjugation bridges, at the material level, the design of highperformance organic EO (OEO) materials requires that the molecular properties of chromophores such as dipole moment $(\mu)$, stability and solubility be optimized rather than the $\beta$ values alone. For the electric field poling process as an essential step in materials processing, it remains less clearly understood how the molecular $\mu \beta$ values of chromophores can be effectively translated into large EO coefficients ( $r_{33}$ values) in poled films and devices. ${ }^{10-16}$

Specifically, the first hyperpolarizability of push-pull chromophores is a third rank tensor that can be described using a $3 \times 3 \times 3$ matrix, which is usually dominated by one diagonal tensor term $\left(\beta_{x x x}\right)$ along the donor-acceptor axis (denoted as the $x$-axis). However, in the experimental measurement of poled OEO films, instead of $\beta_{x x x}$, it is the $\beta_{\mu}$, the magnitude of the irreducible part of the $\beta$ tensor along the dipole vector of chromophores, that is responsible for the macroscopic EO activity ( $r_{33}$ values) of materials. ${ }^{17-19}$ This is similar to the data analysis of molecular hyperpolarizability measurement in solution through electric field induced second harmonic generation (EFISH). The recent 
development of large $\beta$ chromophores is mostly based on pushpull structures with extended $\pi$-conjugation and geometry optimized 3D conformation of the donor and acceptor groups. These molecules would possess unique components of hyperpolarizability tensor and ground-state dipole moment vector in substantial magnitude, and thus they cannot be treated as a simple one-dimensional charge-transfer system for $\mu \beta$ analysis. There are a few studies calculating the $\beta_{\mu}, \beta_{x x x}$, and total $\beta$ tensor, which can be significantly different in magnitude by up to $50 \% .{ }^{18,19}$ However, definitive experimental evidence substantiating the critical role of $\beta_{\mu}$ over that of the $\beta_{x x x}$ term to the bulk NLO response of materials is still lacking, especially for the characterization of poled OEO films containing large $\beta$ chromophores.

To better understand how the $\beta_{\mu}$ values of push-pull chromophores are related to the $r_{33}$ values of materials, we hereby report the rational molecular design of thiophene-bridge-based pushpull chromophores by alternating the sequence of $\pi$-conjugation units to boost the EO activities of poled polymers (Chart 1). Using thiophene as a conjugation unit has long been studied for the design and synthesis of thermally stable push-pull chromophores for second-order NLO. ${ }^{20-29}$ A representative example is the dialkylamino-substituted thiophene stilbenes with strong TCF (tricyanovinyldihydrofuran) and $\mathrm{CF}_{3}-\mathrm{TCF}$ acceptors, previously denoted as FTC chromophores. ${ }^{30}$ Compared to the push-pull polyenes, FTC-type chromophores showed blue-shifted absorption
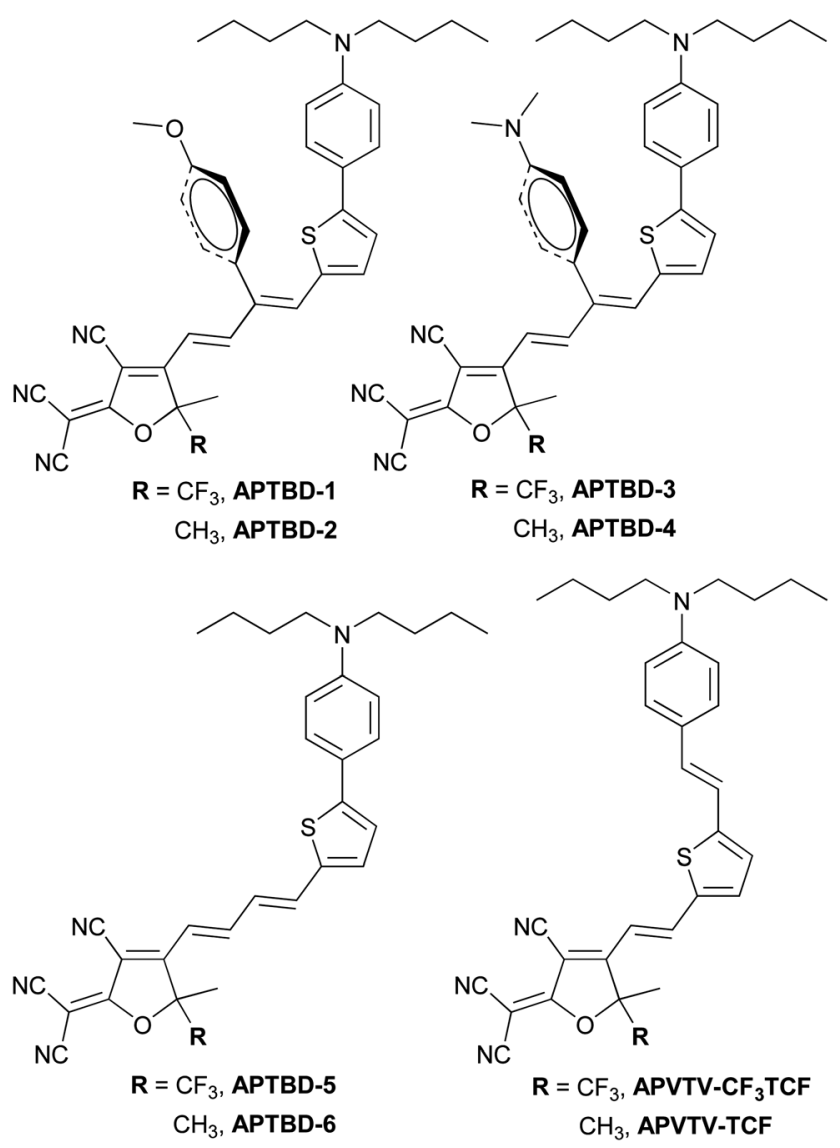

Chart 1 Molecular structures of thiophene-bridge-based push-pull chromophores in this study. in the near-infrared (NIR) region and excellent optical transparency around $1300 \mathrm{~nm}$ and $1550 \mathrm{~nm}$, respectively, applicable to both the second and third telecom windows for low loss waveguides. However, the reported certified $r_{33}$ values of poled polymer waveguides containing these chromophores are mostly in the range of 40-60 $\mathrm{pm} \mathrm{V}^{-1}$ at the telecom wavelengths, which were suboptimal leaving room for improvement. ${ }^{31-33}$

Through a systematic study of the linear and EO properties of this series of new chromophores, the relationship between molecular and bulk NLO responses has been analyzed and compared with thiophene-stilbene-based chromophores as one of the benchmarks for EO devices. Through density functional theory (DFT) calculations, we find that alternating the sequence of $\pi$-conjugation units of thiophene-bridge-based chromophores can significantly affect the shape and dipole moment components of molecules, which are the key molecular parameters in improving the $\mu \beta_{\mu}$ product of chromophores for higher $r_{33}$ values of poled polymers. We also find unexpected results of orthometric site-isolators in affecting the properties of these chromophores in terms of solubility, NLO property, and geometric isomerism, which is highly dependent on the strength and structural asymmetry of TCF and $\mathrm{CF}_{3}$-TCF acceptors. Our study leads to remarkable performance improvement of these chromophores in their molecular hyperpolarizabilities and very effective translation to large $r_{33}$ values up to $106 \mathrm{pm} \mathrm{V}^{-1}$ at $1304 \mathrm{~nm}$ in poled polymers. Furthermore, it demonstrates that optimizing the $\mu \beta_{\mu}$ product (instead of $\mu \beta_{x x x}$ ) as the chromophore figure of merit and increasing the loading density of chromophores are critically important in realizing the large bulk NLO response of OEO materials for performance breakthrough.

\section{Results and discussion}

\section{Synthesis and structural characterization}

Scheme 1 shows the synthetic routes for new push-pull chromophores based on the 4-[5-(4-dibutylamino-phenyl)-thiophen-2-yl]buta-1,3-dienyl donor-bridge component (APTBD) and strong TCF or $\mathrm{CF}_{3}$-TCF acceptors. A generic forward scheme has been devised to synthesize the key intermediates of donor- $\pi$-bridge aldehydes, optionally being incorporated with the site-isolation groups, which were further condensed with TCF and $\mathrm{CF}_{3}-\mathrm{TCF}$ acceptors, respectively, to afford six new chromophores (APTBD1 to APTBD-6) in good yields.

The donor precursor dibutyl-(4-thiophen-2-yl-phenyl)-amine (N-3) was prepared by a simple, two-step synthesis of bromination and Stille coupling. Compound $\mathbf{N}-3$ was then converted to the aldehyde intermediate $\mathbf{N}-\mathbf{4}$ through Vilsmeier formylation, followed by base-catalyzed Knoevenagel condensation with 4 -substituted phenylacetonitriles ${ }^{34}$ and reduction with diisobutylaluminium hydride (DIBAL-H) to produce the APTBD donorbridge aldehydes $\mathbf{N}-\mathbf{7}$ and $\mathbf{N}-\mathbf{8}$ bearing the site-isolators of 4'-methoxyphenyl (MeOPh) and 4'-dimethylaminophenyl (DMA) groups, respectively. Alternately, the lithiation of $\mathbf{N}-3$, followed by electrophilic quenching with 3-dimethylaminoacrolein, produced the pristine APTBD bridge aldehyde $\mathbf{N}-\mathbf{9}$ without the isolation 

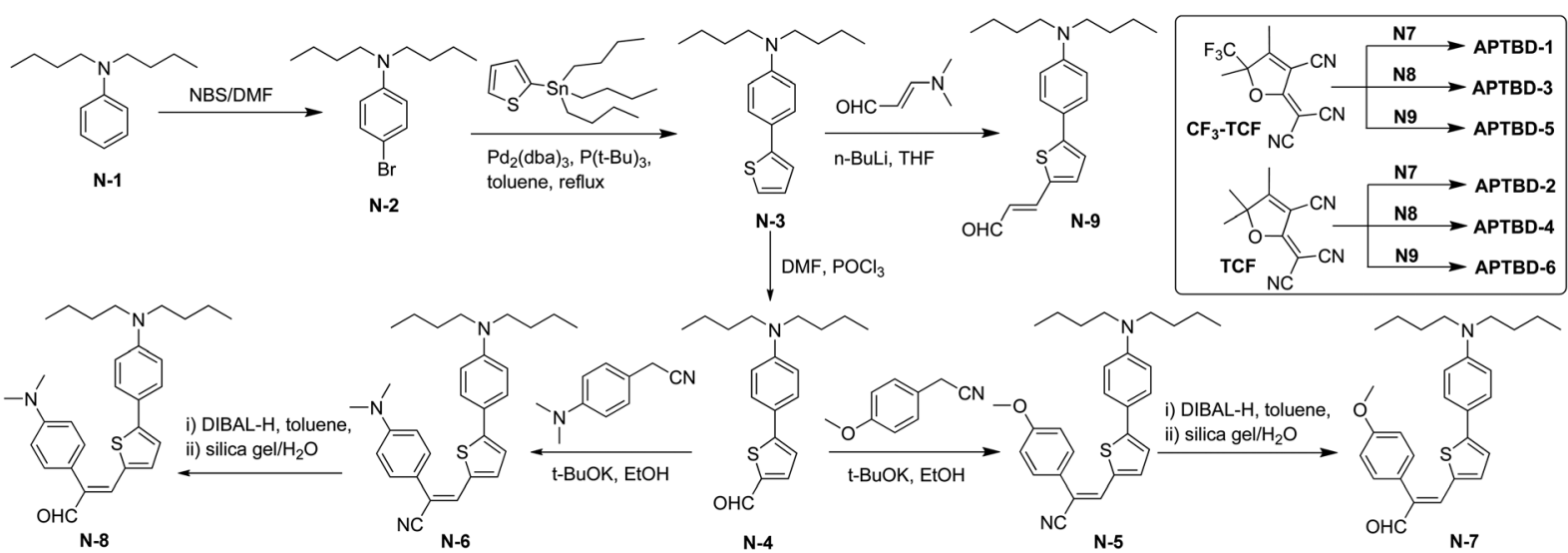

Scheme 1 Synthesis of thiophene-bridge-based push-pull chromophores in this study.

groups. Two benchmark chromophores based on the traditional $\pi$-conjugation structure of dialkylamino-substituted thiophene stilbenes, namely APVTV-CF $\mathbf{F}_{3} \mathbf{T C F}$ and APVTV-TCF, were also included for the comparison study.

All of the prepared chromophores were fully characterized by ${ }^{1} \mathrm{H}$ NMR, ${ }^{13} \mathrm{C}$ NMR, HRMS, UV-vis-NIR spectroscopy, and TGA as reported in the ESI. $\dagger$ A careful analysis of ${ }^{1} \mathrm{H}$ NMR spectra for these chromophores revealed an all-trans configuration of the protons of the olefinic backbones, except that APTBD-6 with the TCF acceptor contains a cis/trans isomer mixture with a percentage ratio of $13 \%: 87 \%$. It suggests that the introduction of rigid isolation groups and the use of a stronger $\mathrm{CF}_{3}$-TCF acceptor would facilitate the formation of the all-trans rigid configuration of this series of $\mathrm{D}-\pi-\mathrm{A}$ chromophores.
From TGA, these new APTBD-series chromophores show very high decomposition temperatures $\left(T_{\mathrm{d}}\right)$ greater than $270{ }^{\circ} \mathrm{C}$, which is nearly $30{ }^{\circ} \mathrm{C}$ higher than those of APTPV chromophores. ${ }^{35}$ The comparison suggests that the relocation of $\pi$-conjugation units can improve the intrinsic stability of chromophores by impeding the potential pathways for bimolecular reaction mechanisms of thermal decomposition.

\section{Solvatochromic properties of chromophores}

In order to study the effect of $\pi$-bridge alternation on the intramolecular charge-transfer (ICT) of chromophores, the UV-vis-NIR absorption spectra of these chromophores were measured in a series of solvents with different dielectric constants. As shown in
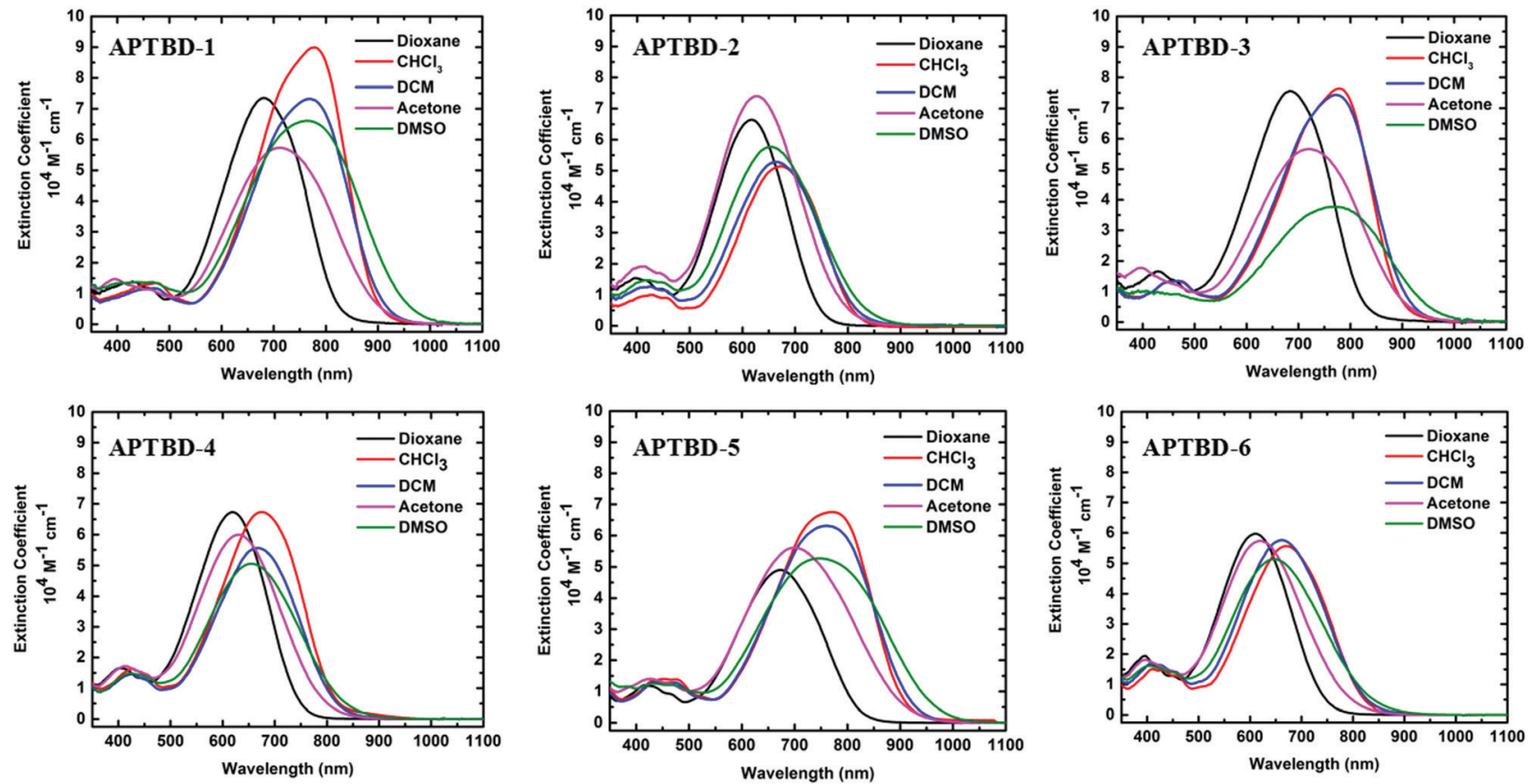

Fig. 1 UV-vis-NIR spectra of APTBD-series chromophores recorded in different solvents of variable dielectric constants at $25^{\circ} \mathrm{C}$. 
Fig. 1, all these chromophores exhibit highly solvatochromic ICT absorption bands in the visible to NIR region. Chromophores APTBD-1, APTBD-3, and APTBD-5 have the maximum absorption $\left(\lambda_{\max }\right)$ around $775 \mathrm{~nm}$ in chloroform, red-shifted by $100 \mathrm{~nm}$ than those of APTBD-2, APTBD-4, and APTBD-6, respectively. It reflects a much stronger electron-withdrawing power of $\mathrm{CF}_{3}$-TCF than that of simple TCF. It is worth noting that the solvatochromism of APTBD-5 is very similar to its constitutional isomer APVTV$\mathbf{C F}_{3} \mathbf{T C F}$ (Fig. S50, ESI $\dagger$ ), suggesting a minor effect of $\pi$-bridge alternation on the ICT of chromophores.

The introduction of the site-isolating $\mathrm{MeOPh}$ group for APTBD-1 and APTBD-2, or the DMA group for APTBD-3 and APTBD-4 contributed to minor red-shift of their $\lambda_{\text {max }}$ values in the solution, as compared to the unsubstituted chromophores with the same donor and acceptor groups. The preliminary analysis of solvatochromism data suggests that the substitution of isolation groups to the $\pi$-conjugation bridge does not interfere with the efficient charge-transfer property of APTBD-series chromophores. Instead, the site-isolation groups can increase the rigidity of the $\pi$-conjugation bridge, leading to increased molar extinction coefficients of chromophores, especially for APTBD-1 and APTBD-2.

\section{Electrochemical properties and density functional theory (DFT)} study of chromophores

As shown in Fig. 2, all chromophores exhibited one reversible oxidative wave with a half-wave potential, $E_{1 / 2}=0.5\left(E_{\mathrm{ox}}+E_{\mathrm{red}}\right)$, versus ferrocene/ferrocenium $\left(\mathrm{Fc} / \mathrm{Fc}^{+}\right)$at about $0.26 \mathrm{~V}$ for APTBD1, 0.23 V for APTBD-2 and APTBD-3, 0.20 V for APTBD-4, 0.27 V for APTBD-5 and $0.24 \mathrm{~V}$ for APTBD-6. The gradual decrease of onset oxidation potential in the series indicates slightly increased electron-donating strength from two unsubstituted chromophores (APTBD-5 and APTBD-6), to two substituted with the MeOPh group (APTBD-1 and APTBD-2), and further to two substituted with the DMA group (APTBD-3 and APTBD-4), for two series of chromophores containing $\mathrm{CF}_{3}-\mathrm{TCF}$ and TCF acceptors, respectively.

A quasi-reversible reduction wave was also recorded for chromophores, and their HOMO and LUMO levels were then calculated from their corresponding oxidation and reduction potentials. We found that the HOMO levels of these chromophores were at around $-5.0 \mathrm{eV}$ to $-5.07 \mathrm{eV}$, typical for NIR absorbing $\mathrm{D}-\pi-\mathrm{A}$ chromophores containing the 4-N,N-dialkylaminophenyl donors. Meanwhile, the corresponding LUMO levels of these chromophores were located at around $-4.0 \mathrm{eV}$ for APTBD-1, APTBD-3, and APTBD-5, and at around $-3.8 \mathrm{eV}$ for APTBD-2, APTBD-4, and APTBD-6. The results are consistent with the solvatochromic study, reiterating the stronger electron-withdrawing power of $\mathrm{CF}_{3}-\mathrm{TCF}$ than that of TCF.

To obtain a better understanding of these molecules, the geometrical optimization and orbital distribution of ground state for these molecules were conducted using the Gaussian 09 package at the level of B3LYP/6-31G(d,p) (Fig. 3). All molecules were assumed to be in a trans configuration, and their geometries were optimized in the gas phase and given solvents. It is shown that the electronic distribution of HOMOs is delocalized
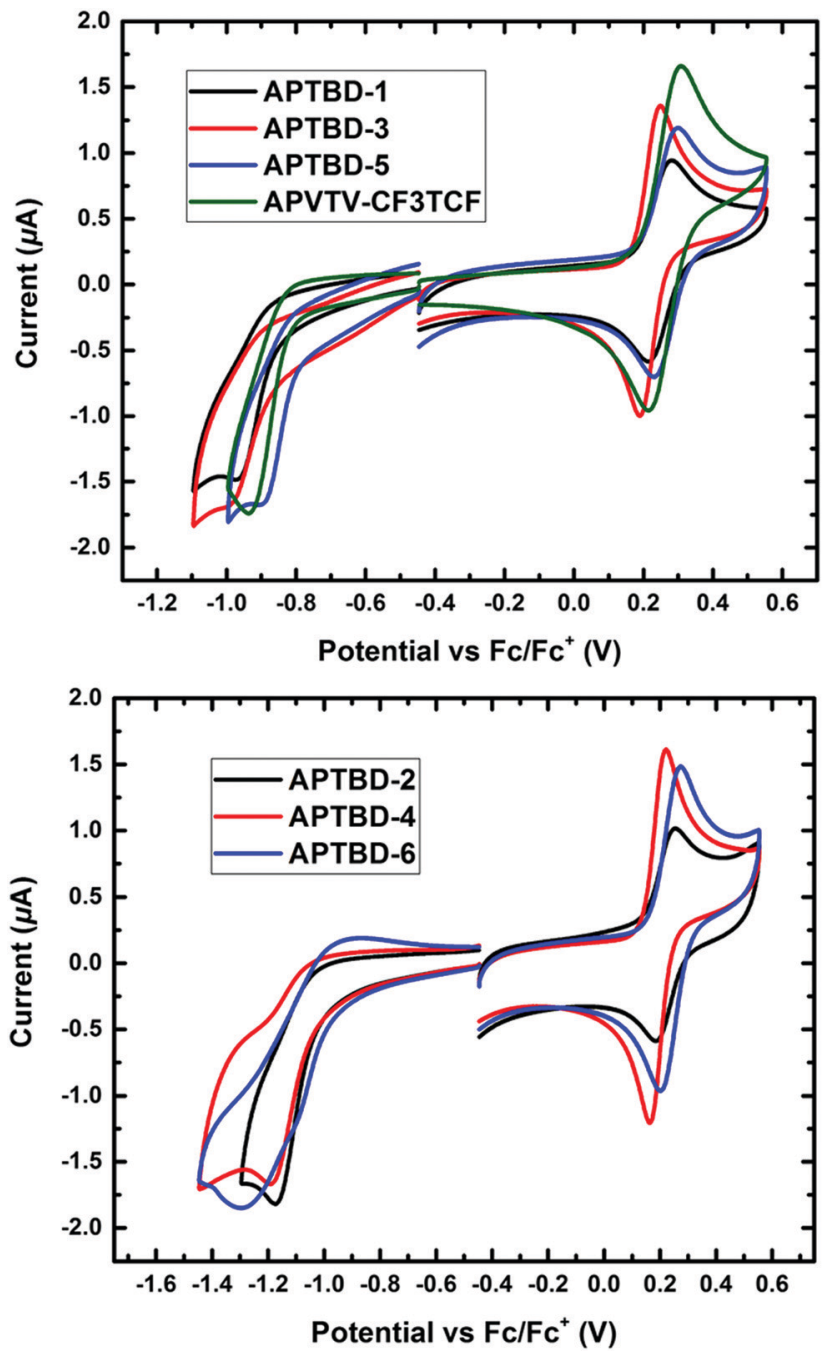

Fig. 2 Cyclic voltammetry (CV) measurements of chromophores recorded in dichloromethane solutions containing $0.1 \mathrm{M} \mathrm{Bu}_{4} \mathrm{NPF}_{6}$ supporting electrolyte.

over the (4-dibutylamino-phenyl)-thiophen-2-yl moiety, whereas the LUMOs are mainly constituted by the dienyl acceptor moieties. The primary $\mathrm{D}-\pi-\mathrm{A}$ frameworks of all chromophores are highly coplanar, while the APTBD-series chromophores give smaller bending angles from the (4-dibutylamino-phenyl)thiophen-2-yl moiety to the dienyl acceptors than the APVTVbased chromophores. For chromophores (APTBD-1 to APTBD-4) with isolation groups, the optimized ground-state geometries of chromophores show that the MeOPh or DMA groups be orthometrically attached to the middle of D- $\pi-$ A structures (Fig. S54, $\mathrm{ESI} \dagger)$. The dihedral angles between the phenyl ring of MeOPh or DMA groups and the $\mathrm{D}-\pi-\mathrm{A}$ plane are around $85^{\circ}$, and interestingly minor participation could be seen from the substitution groups to their HOMOs.

Overall, the trend in the changes of HOMO and LUMO energy levels for these chromophores is consistent from DFT analysis to CV measurements and agrees well with the data of optical bandgap ( $\lambda_{\text {onset }}$ and $E_{\mathrm{g}}$ ) from the optical absorption of chromophores. Such analyses can be used as a guide to explain 

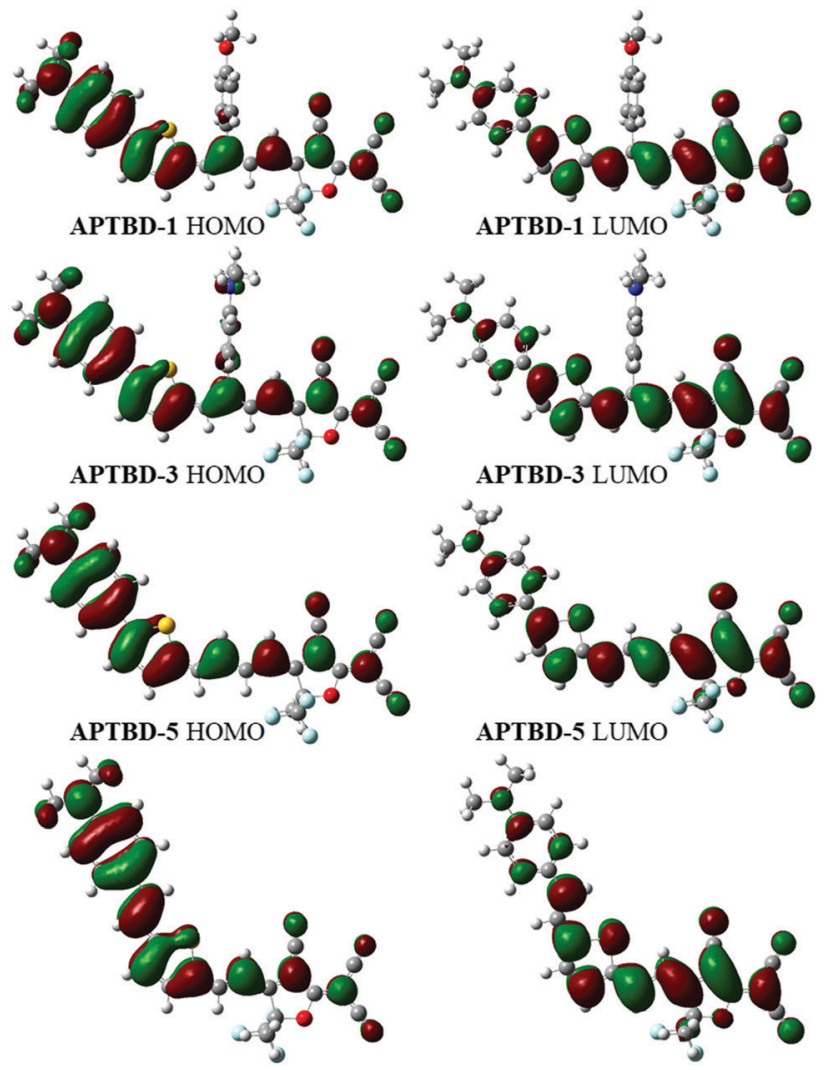

APVTV-CF F $_{3}$ CF HOMO

APVTV-CF 3 TCF LUMO

Fig. 3 Optimized structures and frontier molecular orbital diagrams of chromophores with the $\mathrm{CF}_{3}$-TCF acceptor by DFT with the Gaussian basis set of $B 3 L Y P / 6-31 G(d, p)$ in gas phase.

the results in the study of solvatochromic and electrochemical properties of chromophores (Table 1).

Study of the poling-induced birefringence, EO properties and rigid oriented gas model analysis for poled films

Following the standard formulation and processing protocols of EO polymers, the optical quality thin films of guest-host polymers containing a loading level of $20 \mathrm{wt} \%$ of chromophores were prepared, except only $10 \mathrm{wt} \%$ for APTBD-6 due to its poor solubility. Poly(styrene-co-methyl methacrylate) (P(S-co-MMA)) is selected as the host polymer due to its excellent optical and

Table 1 The analysis of the HOMO and LUMO levels and bandgap by DFT and $\mathrm{CV}$

\begin{tabular}{|c|c|c|c|c|c|c|}
\hline & \multicolumn{3}{|c|}{ DFT (gas phase) } & \multicolumn{3}{|c|}{$\mathrm{CV}$ in $\mathrm{CH}_{2} \mathrm{Cl}_{2}$} \\
\hline & $\begin{array}{l}\text { HOMO } \\
(\mathrm{eV})\end{array}$ & $\begin{array}{l}\text { LUMO } \\
(\mathrm{eV})\end{array}$ & $\begin{array}{l}E_{\mathrm{g}} \\
(\mathrm{eV})\end{array}$ & $\begin{array}{l}\text { HOMO } \\
(\mathrm{eV})\end{array}$ & $\begin{array}{l}\text { LUMO } \\
(\mathrm{eV})\end{array}$ & $\begin{array}{l}E_{\mathrm{g}} \\
(\mathrm{eV})\end{array}$ \\
\hline APTBD-1 & -5.26 & -3.20 & 2.06 & -5.06 & -3.97 & 1.09 \\
\hline APTBD-2 & -5.21 & -3.05 & 2.16 & -5.03 & -3.79 & 1.24 \\
\hline APTBD-3 & -5.21 & -3.14 & 2.07 & -5.03 & -3.97 & 1.06 \\
\hline APTBD-4 & -5.14 & -2.97 & 2.16 & -5.00 & -3.78 & 1.22 \\
\hline APTBD-5 & -5.38 & -3.35 & 2.04 & -5.07 & -4.02 & 1.05 \\
\hline APTBD-6 & -5.32 & -3.19 & 2.13 & -5.04 & -3.83 & 1.21 \\
\hline APVTV-CF ${ }_{3}$ TCF & -5.32 & -3.32 & 2.00 & -5.06 & -4.02 & 1.04 \\
\hline
\end{tabular}

dielectric properties and good compatibility with TCF-based chromophores. The modest number density is used in the study, and the films were poled under an electric field of $100 \mathrm{~V} \mu \mathrm{m}^{-1}$. This poling protocol is applied such that all films can be poled efficiently to the optimal temperature around the glass transition temperature $\left(T_{\mathrm{g}}\right)$ of polymers, which is essential for systematic structure-property relationship studies.

The $r_{33}$ values and poling-induced optical birefringence of all poled films were measured in slab waveguide geometry on a widely used prism-coupler system Metricon 2010/M. Without any encapsulation, the poled films showed excellent temporal stability in the handling of slab waveguide measurements under ambient conditions. The $r_{33}$ values of poled films were calculated by measuring the change of refractive indices under the application of modulation field $E$ according to the Pockels effect: ${ }^{36,37}$

$$
\Delta\left(\frac{1}{n_{\mathrm{TM}}^{2}}\right)=r_{33} E
$$

The order parameters of poled films were calculated from the refractive indices of $n_{\mathrm{TE}}$ and $n_{\mathrm{TM}}$, and used as a measure of chromophore alignment in the poled films. A Sellmeier fit of refractive indices was conducted on the values of refractive indices at $1304 \mathrm{~nm}$ and $1541 \mathrm{~nm}$ using a simple model of absorption and dispersion, in which the refractive index $(n)$ is assumed to have contributions from both a non-resonant background $n_{0}$ and the two-level charge transfer resonance at frequency $\nu_{0}$ in $\mathrm{cm}^{-1} \cdot{ }^{38}$ Thus, at an operating frequency of $\nu$, we have

$$
n=n_{0}+\frac{A}{\nu_{0}^{2}-\nu^{2}}
$$

The order parameter $\Phi$ for poled EO films is given by

$$
\Phi=\frac{\delta_{\mathrm{TM}}-\delta_{\mathrm{TE}}}{\delta_{\mathrm{TM}}+2 \delta_{\mathrm{TE}}}
$$

where $\delta_{\mathrm{TM}}=n_{\mathrm{TM}}-n_{0}$ and $\delta_{\mathrm{TE}}=n_{\mathrm{TE}}-n_{0}$. The obtained results for order parameters and $r_{33}$ values are summarized in Table 2 . Since the refractive indices are measured at two wavelengths away from the main ICT absorption peak of chromophores, this characterization technique is more reliable than the absorbance measurement in evaluating the poling efficiency of high vis-NIR absorbing poled EO films.

With the modest loading level of chromophores, it is reasonable to use a rigid oriented gas model (ROGM) to analyze the orientational order of dipolar chromophores from poling-induced refractive index changes of poled EO films. This is a simplified semi-empirical model that laid a solid foundation for the field of poled polymers. It encompasses a variety of molecular and material parameters for reliable analyses without overly involving extensive computational and experimental analyses. It has been used in our research to achieve consistent results. According to ROGM, we correlate the measured $\Phi$ values as the second-rank axial order parameter to the non-centrosymmetric order parameter $\left\langle\cos ^{3} \theta\right\rangle$ of poled films, where $\theta$ is the angle between the permanent dipole moment of chromophores and applied electric 
Table 2 Summary of the poling-induced birefringence, EO properties and ROGM analysis for poled films

\begin{tabular}{|c|c|c|c|c|c|c|c|}
\hline Chromophore & $N\left(10^{20} \mathrm{~cm}^{-3}\right)$ & $\lambda_{\max } / \mathrm{nm}$ in films & $n_{\mathrm{TE}} / n_{\mathrm{TM}}$ at $1304 \mathrm{~nm}$ & $r_{33}$ at $1304 \mathrm{~nm}\left(\mathrm{pm} \mathrm{V}^{-1}\right)$ & $r_{33} / N$ & $\Phi$ & $\beta_{\mu}(-\omega ; \omega, 0)\left(10^{-30} \mathrm{esu}\right)$ \\
\hline APTBD-1 & 1.94 & 725 & $1.5854 / 1.6291$ & 53.8 & 27.7 & 0.160 & 2284 \\
\hline APTBD-3 & 1.90 & 737 & $1.5783 / 1.6107$ & 53.4 & 28.1 & 0.107 & 2346 \\
\hline APTBD-5 & 2.30 & 716 & $1.6034 / 1.6632$ & 78.9 & 34.3 & 0.146 & 2629 \\
\hline APTBD-5 & 2.87 & 719 & $1.6149 / 1.7092$ & 106.1 & 36.9 & 0.190 & 2646 \\
\hline APVTV-CF ${ }_{3}$ TCF & 2.30 & 722 & $1.5962 / 1.6419$ & 60.8 & 26.4 & 0.098 & 2405 \\
\hline APTBD-2 & 2.11 & 648 & $1.5790 / 1.5937$ & 26.5 & 12.6 & 0.066 & 1289 \\
\hline APTBD-4 & 2.06 & 654 & $1.5767 / 1.6079$ & 28.3 & 13.7 & 0.142 & 1056 \\
\hline APTBD-6 & 1.27 & 639 & $1.5494 / 1.5632$ & 18.1 & 14.3 & 0.072 & 1365 \\
\hline
\end{tabular}

field. ${ }^{39-41}$ Overall, the poled films of these chromophores give a high poling-induced polar order, comparatively large but highly variable $r_{33}$ values at $1304 \mathrm{~nm}$.

According to ROGM, the $r_{33}$ value of poled films is given by

$$
r_{33}=2 N f_{0} f_{\lambda}^{2} \beta_{\mu}(-\omega ; \omega, 0)\left\langle\cos ^{3} \theta\right\rangle / n_{\mathrm{TM}}{ }^{4}
$$

where $\beta_{\mu}(-\omega ; \omega, 0)$ is the vector part of $\beta$ tensors along the dipole moment direction of chromophores, $f_{0}$ is the zero-frequency local field factor for the electric field (or Onsager local field correction), and $f_{\lambda}$ is the local field factor for the lightwave field (or Lorentz-Lorentz local field correction), respectively. ${ }^{39-41} \mathrm{We}$ follow the convention of local field factors according to

$$
\begin{gathered}
f_{0}=\varepsilon\left(n^{2}+2\right) /\left(n^{2}+2 \varepsilon\right) \\
f_{\lambda}=\left(n^{2}+2\right) / 3
\end{gathered}
$$

where $\varepsilon$ is the dielectric constant of guest-host polymers in $\mathrm{P}(\mathrm{S}-$-co-MMA) from the impedance analyzer.

After quantifying all the key parameters including $r_{33}$ values, refractive indices, loading density, local field factors, and polar order parameters $\left(\left\langle\cos ^{3} \theta\right\rangle\right)$, our studies indicate that APTBDbased chromophores exhibit large $\beta_{\mu}(-\omega ; \omega, 0)$ values at $1304 \mathrm{~nm} .+$ For three chromophores with TCF acceptors, the values are $1056 \times 10^{-30}$ esu for APTBD-4, $1289 \times 10^{-30}$ esu for APTBD-2, and $1365 \times 10^{-30}$ esu for APTBD-6. ${ }^{39}$ The values are nearly doubled for chromophores with a stronger $\mathrm{CF}_{3}-\mathrm{TCF}$ acceptor, around $2300 \times 10^{-30}$ esu for APTBD-1 and APTBD-2, and $2629 \times 10^{-30}$ esu for APTBD-5. The $\beta_{\mu}(-\omega ; \omega, 0)$ values of APTBD-series chromophores are among the highest non-resonant values for dipolar chromophores with the same conjugation length and donor/acceptor structures. At the given loading level of $20 \mathrm{wt} \%$ and poling condition, their $r_{33}$ values, up to $53.4 \mathrm{pm} \mathrm{V}^{-1}$ to $78.9 \mathrm{pm} \mathrm{V}^{-1}$ for chromophores with the $\mathrm{CF}_{3}$-TCF acceptor, are significantly higher than those from FTC-type chromophores in poled slab waveguide geometry by the EO measurement of the prism coupling system.

We found that our analyses of $\beta$ values from poled films agree reasonably with reported results for similar compounds in the solutions by electric-field-induced second-harmonic generation (EFISH) or hyper-Rayleigh scattering (HRS). It reiterates that our EO characterization and quantitative structure-property relationship analysis in guest-host solid-solid solutions is highly

\# The values of hyperpolarizabilities $(\beta)$ in MKS unit are converted to the values in electrostatic units (esu) according to the unit conversion: 1 statvolt ${ }^{-1} \mathrm{~cm}^{4}=$ $4.18888 \times 10^{-10} \mathrm{~m}^{4} \mathrm{~V}^{-1}$. applicable to evaluate the molecular and materials' NLO properties at the off-resonance wavelengths. ${ }^{39-42}$

It has been suggested that the relative merits of NLO chromophores are consistently predictable with common DFT methods. ${ }^{17-19}$ To investigate the critical role of $\beta_{\mu}$ over that of the $\beta_{x x x}$ term to the bulk NLO response of materials, we select two chromophores that are constitutional isomers, namely APTBD-5 and APVTV-CF $\mathbf{B}_{\mathbf{3}} \mathbf{T C F}$, for further investigation. We noticed that, at a loading density of $2.30 \times 10^{20} \mathrm{~cm}^{-3}$, the $r_{33}$ value of poled films with APTBD-5 was up to $78.9 \mathrm{pm} \mathrm{V}^{-1}$ at $1304 \mathrm{~nm}$ and improved by $30 \%$ over that of APVTV-CF $\mathbf{C}_{\mathbf{3}} \mathbf{T C F}$. Based on the fully optimized geometry of chromophores in the gas phase, we calculated their static first hyperpolarizabilities at the cam-B3LYP level by employing the split valence $6-31 \mathrm{G}(\mathrm{d}, \mathrm{p})$ basis set using the "polar" keyword in the Gaussian 09 program package, which gives ten of the unique components of hyperpolarizability tensor. For comparison with experiments, various scalar measures of the tensors need to be computed according to

$$
\beta_{\text {total }}=\left(\beta_{x}^{2}+\beta_{y}{ }^{2}+\beta_{z}{ }^{2}\right)^{\frac{1}{2}}
$$

where the $x, y$ and $z$ components of the hyperpolarizability tensor are

$$
\beta_{i}=\beta_{i i i}+\frac{1}{3} \sum_{i \neq j}\left(\beta_{i j j}+\beta_{i j j}+\beta_{j j i}\right), \quad i, j \in(x, y, z)
$$

The static $\beta_{\mu}$ as the vector part of $\beta$ tensors along the dipole moment direction of chromophores was then calculated by

$$
\begin{gathered}
\beta_{\mu}=\sum_{i=x, y, z}\left(\mu_{i} \beta_{i}\right) /\left(\sum_{i=x, y, z} \mu_{i}^{2}\right)^{\frac{1}{2}} \\
\mu_{\text {total }}=\left(\mu_{x}^{2}+\mu_{y}^{2}+\mu_{z}^{2}\right)^{1 / 2}
\end{gathered}
$$

where $\mu_{x}, \mu_{y}$, and $\mu_{z}$ are three components of the ground-state dipole moment vector of chromophores from the geometry optimization. ${ }^{17-19}$ These results are summarized in Table 3.

From the DFT analysis, APTBD-5 possesses a $\mu_{\text {total }}$ of $21.9 \mathrm{D}$ and a $\beta_{\mu}$ of $617.0 \times 10^{-30}$ esu. Its nearly identical $\beta_{\mu}$ and $\beta_{x x x}$ values suggest an excellent overlap between the dominant $\beta$ tensor term and the dipole moment of chromophores, mainly due to the relatively small bending angle along with the majority of its D- $\pi$-A framework. In comparison, APVTV-CF $\mathbf{3}$ TCF has a $\mu_{\text {total }}$ of $20.6 \mathrm{D}$ and a $\beta_{\mu}$ of $572.6 \times 10^{-30} \mathrm{esu}$, the latter being considerably

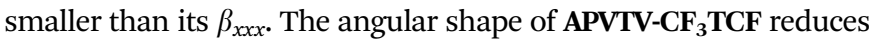


Table 3 Summary and figure-of-merit analysis of components of the ground-state dipole moment vector and static first hyperpolarizability tensors of chromophores by DFT

\begin{tabular}{|c|c|c|c|c|c|c|c|c|c|c|c|}
\hline Chromophore & \multicolumn{4}{|l|}{$\underline{\mu(\mathrm{D})}$} & \multicolumn{5}{|c|}{$\beta\left(\times 10^{-30} \mathrm{esu}\right)$} & $\mu_{\text {total }} \beta_{\mu}\left(\times 10^{-48}\right.$ esu $)$ & $r_{33}$ at $N$ of $2.30 \times 10^{20} \mathrm{~cm}^{-3}$ \\
\hline APTBD-5 & -20.8 & -6.9 & -1.5 & 21.9 & 652.7 & -0.2 & -14.5 & 652.9 & -617.0 & 13523 & 78.9 \\
\hline
\end{tabular}

the overlap between the principle $\beta$ term and $\mu$. The subtle differences in the components of ground-state dipole moment vector of chromophores lead to a substantial improvement of the $\mu_{\text {total }} \beta_{\mu}$ value of $13523 \times 10^{-48}$ esu for APTBD-5 over that of $11774 \times 10^{-48}$ esu for APVTV-CF ${ }_{3}$ TCF. The DFT analysis follows the trend of experimental results reasonably well and clearly verifies the $\mu_{\text {total }} \beta_{\mu}$ product as the figure of merit in determining the NLO activity of chromophores in poled polymers.

One of our initial motivations in the design of chromophores APTBD-1 to APTBD-4 is to follow our earlier study in a shorter D- $\pi-\mathrm{A}$ structure to test the feasibility of such site-isolation groups in large D- $\pi-\mathrm{A}$ chromophores for performance improvement. ${ }^{15,26}$ However, we obtained mixed results for APTBD-series chromophores with isolation groups. For chromophores with the TCF acceptor, we found that APTBD-2 and APTBD-4 chromophores showed higher solubility and better compatibility with the host polymer for higher $r_{33}$ values than that of APTBD-6. In sharp contrast, all chromophores with the $\mathrm{CF}_{3}$-TCF acceptor, namely APTBD-1, APTBD-3, and APTBD-5, are highly soluble in organic solvents and compatible with the host polymer up to a high loading level. The comparison underscores the critical role of the trifluoromethyl group in chromophores containing the $\mathrm{CF}_{3}$-TCF acceptor over the TCF-based analogs, not only increasing the electron-withdrawing power of the acceptor for higher $\beta$ but also creating a small $z$-component $\left(\mu_{z}\right)$ of dipole moment. It can effectively prevent close packing of $\mathrm{D}-\pi-\mathrm{A}$ chromophores with predominant $\mu_{x}$ and $\mu_{y}$ components. ${ }^{43-47}$

As shown earlier, the introduction of the site-isolating MeOPh group for APTBD-1 and APTBD-2, or the DMA group for APTBD-3 and APTBD-4 contributed to a slight red-shift of their $\lambda_{\max }$ values and higher molar extinction coefficients in the solutions, as compared to the unsubstituted chromophores APTBD-5 and APTBD-6, respectively. However, in the study of poled films, the unsubstituted chromophores gave better EO properties than their substituted ones. We speculate that the performance of these chromophores may be affected by some factors such as condensed phase effects and vibrational contributions. This is beyond the scope of this work and may deserve further study.

Finally, we found that APTBD-5 is highly compatible with the host polymer $\mathrm{P}(\mathrm{S}-c o-\mathrm{MMA})$ through solution processing, and the optical bandgap of solid films is around $1000 \mathrm{~nm}$, which suffice for achieving high optical transparency at broad NIR wavelengths (Fig. S54, ESI $\dagger$ ). At a higher loading level of $2.87 \times 10^{20} \mathrm{~cm}^{-3}$ with efficient poling, very large $r_{33}$ values, up to $106.1 \mathrm{pm} \mathrm{V}^{-1}$ with $n_{\mathrm{TE}} / n_{\mathrm{TM}}$ of $1.6149 / 1.7092$ at $1304 \mathrm{~nm}$ and $88.7 \mathrm{pm} \mathrm{V}^{-1}$ with $n_{\mathrm{TE}} / n_{\mathrm{TM}}$ of $1.6000 / 1.6725$ at $1541 \mathrm{~nm}$, have been achieved in the poled films. The values are among the highest reported non-resonant $r_{33}$ values at two major telecom wavelengths in poled slab waveguides and comparable to the performance from push-pull tetraene chromophores. ${ }^{33,37,43}$ Given the blue-shifted $\lambda_{\max }$ at $719 \mathrm{~nm}$ and the band edge of the APTBD-5 5 chromophore in the series, the results are extremely encouraging for the future development of low-loss waveguide modulators at both the second and third telecom windows.

\section{Conclusions}

We report the synthesis and systematic study of the linear and EO properties of new thiophene-bridge-based push-pull chromophores by alternating the sequence of $\pi$-conjugation units to boost the EO activities of poled polymers. The relationship between molecular and bulk NLO responses of these chromophores has been analyzed and compared with thiophene-stilbenebased chromophores as one of the benchmarks for EO devices. Through ROGM and DFT calculations, we find that alternating the sequence of $\pi$-conjugation units of thiophene-bridge-based chromophores can significantly affect the dipole moment components of molecules, which are the key molecular parameters in improving the $\mu_{\text {total }} \beta_{\mu}$ product of chromophores for higher $r_{33}$ values of poled polymers. We also obtained counter-intuitive results of orthometric site-isolators in affecting the properties of these chromophores in terms of solubility, NLO property, and geometric isomerism, which is highly dependent on the strength and structural asymmetry of TCF and $\mathrm{CF}_{3}-\mathrm{TCF}$ acceptors.

Our study leads to the remarkable performance of new APTBD-based chromophores in their molecular hyperpolarizabilities and very effective translation to large $r_{33}$ values up to $106.1 \mathrm{pm} \mathrm{V}^{-1}$ at $1304 \mathrm{~nm}$ and $88.7 \mathrm{pm} \mathrm{V}^{-1}$ at $1541 \mathrm{~nm}$ in poled polymers, which is over $30 \%$ better than the optimal results from thiophene-stilbene-based chromophore isomers. It demonstrates that optimizing the $\mu_{\text {total }} \beta_{\mu}$ product as the figure of merit of the chromophore and increasing their loading density are critically important in realizing the large bulk NLO response of OEO materials for photonic applications.

\section{Experimental section}

\section{Materials and methods}

All chemicals were purchased from Energy Chemical or Aldrich and used as received unless otherwise mentioned. High-resolution mass spectrometry (HRMS) was performed using a Thermo Scientific Q Exactive mass spectrometer. For the formulation of EO polymers, the solvents dibromomethane (DBM) and 1,1,2trichloroethane (TCE) were distilled prior to use. The cyclic voltammetric data were measured using an Electrochemical 
Analyzer (CHI 750) with $\mathrm{A}_{\mathrm{g}} / \mathrm{A}_{\mathrm{g}} \mathrm{Cl}$ as the reference electrode, a platinum wire as the working electrode, platinum gauze $(5 \times 5 \times 0.3 \mathrm{~mm})$ as the counter electrode and $0.1 \mathrm{M}$ tetrabutylammonium hexafluorophosphate (TBAPF) as the electrolyte in dichloromethane. Thermogravimetric analyses (TGA) were carried out on a PerkinElmer STA 6000 Simultaneous Thermal Analyzer at a heating rate of $20{ }^{\circ} \mathrm{C} \mathrm{min}^{-1}$. The films used in absorption spectrum and EO measurements were spin-coated on glass or ITO glass substrates with SPIN-PROCESS CONTROLLER. The dielectric constants of EO films were measured at low frequencies using a KEYSIGHT E4990A Impedance Analyzer. The UV-vis-NIR spectra of chromophores were recorded using an Ultra-Violet Visible Scanning Spectrophotometer (Shimadzu 1700) and an Ultra-Violet Visible Near Infra-red Spectrophotometer with Integrating Sphere (PE Lambda 750). DFT calculations using the Gaussian 09 package were carried out at the level of B3LYP/6$31 \mathrm{G}(\mathrm{d}, \mathrm{p})$ for ground-state geometry optimization and CAM-B3LYP/ $6-31 G(d, p)$ for static hyperpolarizability calculations.

\section{Poling and measurement of $r_{33}$ values and refractive indices}

For studying the EO property derived from the chromophores, guest-host EO polymers were formulated by mixing chromophores at a given loading density into the host polymer poly(styrene-co-methyl methacrylate) (P(S-co-MMA)) in the solvent DBM (or TCE). The resulting solutions were filtered through a $0.2 \mu \mathrm{m}$ PTFE filter and spin-coated onto indium tin oxide (ITO) glass substrates. After soft baking, the films of doped polymers were baked in a vacuum oven at $60-70{ }^{\circ} \mathrm{C}$ overnight to ensure the complete removal of residual solvent. The thicknesses of the films were measured using a DektakXT Stylus Profiler and further confirmed on the subsequent optical measurement for refractive indices at the wavelengths of $1304 \mathrm{~nm}$ and $1541 \mathrm{~nm}$ using a commercial prism-coupler system (Metricon 2010/M). Then using the Desk V HP Sputter Unit (Denton Vacuum LLC), a thin layer $(\sim 20 \mathrm{~nm})$ of semitransparent gold was sputtered onto the films as the top electrode for contact poling and the subsequent EO coefficient measurement. The electric field poling of films was conducted at a central processor-controlled Mettler FP82 hot stage. The

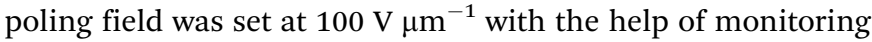
the LTC using a Keithley 2657A Source-Meter Unit. The optimal poling temperatures were around $110-115{ }^{\circ} \mathrm{C}$. After the poling, the refractive indices and $r_{33}$ values of poled films were measured using the attenuated total reflectance (ATR) method in slab waveguide geometry on Metricon 2010/M, in which modulation voltages were applied to the poled films for EO coefficient measurements. The accuracy of the measurement system is verified with standard EO materials including lithium niobate thin film from NANOLN and AJLZ53/P(s-co-MMA) film from our research group.

\section{Conflicts of interest}

There are no conflicts of interest to declare.

\section{Acknowledgements}

The authors acknowledge the financial support from the Fundamental Research Funds for the Fundamental Research Project funding from Shenzhen Science \& Technology Innovation Committee (JCYJ20180507181718203), the Natural Science Foundation of China (21975213), the Research Grants Council (RGC) of Hong Kong (RGC Ref. No. 11306320), internal research supports or initiatives from City University of Hong Kong (9610389, 9680263 and 9610454), and helpful comments and technical assistance from Dr Wen Wang and Dr Jieyun Wu of the University of Electronic Science and Technology of China.

\section{Notes and references}

1 A. J. T. Lou, S. Righetto, C. Barger, C. Zuccaccia, E. Cariati, A. Macchioni and T. J. Marks, Unprecedented Large Hyperpolarizability of Twisted Chromophores in Polar Media, J. Am. Chem. Soc., 2018, 140, 8746-8755.

2 F. Liu, S. Chen, S. Mo, G. Qin, C. Yu, W. Zhang, W. J. Shi, P. Chen, H. Xu and M. Fu, Synthesis of novel nonlinear optical chromophores with enhanced electro-optic activity by introducing suitable isolation groups into the donor and bridge, J. Mater. Chem. C, 2019, 7, 8019-8028.

3 V. Parthasarathy, R. Pandey, P. K. Das, F. Castet and M. Blanchard-Desce, Linear and Nonlinear Optical Properties of Tricyanopropylidene-Based Merocyanine Dyes: Synergistic Experimental and Theoretical Investigations, ChemPhysChem, 2018, 19, 187-197.

4 S. Pascal, Y. A. Getmanenko, Y. Zhang, I. Davydenko, M. H. Ngo, G. Pilet, S. Redon, Y. Bretonnière, O. Maury, I. Ledoux-Rak, S. Barlow, S. R. Marder and C. Andraud, Design of near-infrared-absorbing unsymmetrical polymethine dyes with large quadratic hyperpolarizabilities, Chem. Mater., 2018, 30, 3410-3418.

5 W. Heni, Y. Kutuvantavida, C. Haffner, H. Zwickel, C. Kieninger, S. Wolf, M. Lauermann, Y. Fedoryshyn, A. F. Tillack, L. E. Johnson, D. L. Elder, B. H. Robinson, W. Freude, C. Koos, J. Leuthold and L. R. Dalton, Siliconorganic and plasmonic-organic hybrid photonics, ACS Photonics, 2017, 4, 1576-1590.

6 M. Ayata, Y. Fedoryshyn, W. Heni, B. Baeuerle, A. Josten, M. Zahner, U. Koch, Y. Salamin, C. Hoessbacher, C. Haffner, D. L. Elder, L. R. Dalton and J. Leuthold, High-speed plasmonic modulator in a single metal layer, Science, 2017, 358, 630-632.

7 C. Haffner, D. Chelladurai, Y. Fedoryshyn, A. Josten, B. Baeuerle, W. Heni, T. Watanabe, T. Cui, B. Cheng, S. Saha, D. L. Elder, L. R. Dalton, A. Boltasseva, V. M. Shalaev, N. Kinsey and J. Leuthold, Low-loss plasmonassisted electro-optic modulator, Nature, 2018, 556, 483-486.

8 S. Ummethala, T. Harter, K. Koehnle, Z. Li, S. Muehlbrandt, Y. Kutuvantavida, J. Kemal, P. Marin-Palomo, J. Schaefer, A. Tessmann, S. K. Garlapati, A. Bacher, L. Hahn, M. Walther, T. Zwick, S. Randel, W. Freude and C. Koos, THz-to-optical conversion in wireless communications 
using an ultra-broadband plasmonic modulator, Nat. Photonics, 2019, 13, 519-524.

9 J. D. Witmer, T. P. McKenna, P. Arrangoiz-Arriola, R. Van Laer, E. A. Wollack, F. Lin, A. K. Jen, J. Luo and A. H. SafaviNaeini, A silicon-organic hybrid platform for quantum microwave-to-optical transduction, Quantum Sci. Technol., 2020, 5, 034004.

$10 \mathrm{~J} . \mathrm{Wu}, \mathrm{Z}$. Li, J. Luo and A. K. Y. Jen, High-performance organic second-and third-order nonlinear optical materials for ultrafast information processing, J. Mater. Chem. C, 2020, 8, 15009-15026.

11 M. Li, Y. Li, H. Zhang, S. Wang, Y. Ao and Z. Cui, Molecular engineering of organic chromophores and polymers for enhanced bulk second-order optical nonlinearity, J. Mater. Chem. C, 2017, 5, 4111-4122.

12 J. Liu, G. Xu, F. Liu, I. Kityk, X. Liu and Z. Zhen, Recent advances in polymer electro-optic modulators, RSC Adv., 2015, 5, 15784-15794.

13 R. Tang, S. Zhou, Z. Cheng, G. Yu, Q. Peng, H. Zeng, G. Guo, Q. $\mathrm{Li}$ and $\mathrm{Z}$. Li, Janus second-order nonlinear optical dendrimers: their controllable molecular topology and corresponding largely enhanced performance, Chem. Sci., 2017, 8, 340-347.

14 J. Wu, B. Wu, W. Wang, K. S. Chiang, A. K. Y. Jen and J. Luo, Ultra-efficient and stable electro-optic dendrimers containing supramolecular homodimers of semifluorinated dipolar aromatics, Mater. Chem. Front., 2018, 2, 901-909.

$15 \mathrm{~J}$. Wu, W. Wang, K. Chen and J. Luo, The synthesis of second-order nonlinear optical chromophores with conjugated steric hindrance for electro-optics at $850 \mathrm{~nm}, J$. Mater. Chem. C, 2020, 8, 5494-5500.

16 L. R. Dalton, P. A. Sullivan and D. H. Bale, Electric field poled organic electro-optic materials: state of the art and future prospects, Chem. Rev., 2010, 110, 25-55.

17 C. M. Isborn, A. Leclercq, F. D. Vila, L. R. Dalton, J. L. Bredas, B. E. Eichinger and B. H. Robinson, Comparison of static first hyperpolarizabilities calculated with various quantum mechanical methods, J. Phys. Chem. A, 2007, 111, 1319-1327.

18 A. P. Chafin and G. A. Lindsay, A pattern for increasing the first hyperpolarizability of a push-pull polyene dye as indicated from DFT calculations, J. Phys. Chem. C, 2008, 112, 7829-7835.

19 J. Hung, W. Liang, J. Luo, Z. Shi, A. K.-Y. Jen and X. Li, Rational design using Dewar's rules for enhancing the first hyperpolarizability of nonlinear optical chromophores, J. Phys. Chem. C, 2010, 114, 22284-22288.

20 P. R. Varanasi, A. K.-Y. Jen, J. Chandrasekhar, I. N. N. Namboothiri and A. Rathna, The important role of heteroaromatics in the design of efficient second-order nonlinear optical molecules: Theoretical investigation on push-pull heteroaromatic stilbenes, J. Am. Chem. Soc., 1996, 118, 12443-12448.

21 J. M. Raimundo, P. Blanchard, N. Gallego-Planas, N. Mercier, I. Ledoux-Rak, R. Hierle and J. Roncali, Design and synthesis of push-pull chromophores for second-order nonlinear optics derived from rigidified thiophene-based $\pi$-conjugating spacers, J. Org. Chem., 2002, 67, 205-218.
22 M. He, T. M. Leslie and J. A. Sinicropi, Synthesis of chromophores with extremely high electro-optic activity. 1 . Thiophenebridge-based chromophores, Chem. Mater., 2002, 14, 4662-4668.

23 Y. J. Cheng, J. Luo, S. Hau, D. H. Bale, T. D. Kim, Z. Shi, D. B. Lao, N. M. Tucker, Y. Tian, L. R. Dalton, P. J. Reid and A. K.-Y. Jen, Large electro-optic activity and enhanced thermal stability from diarylaminophenyl-containing high- $\beta$ nonlinear optical chromophores, Chem. Mater., 2007, 19, 1154-1163.

24 H. Zhang, Y. Tian, S. Bo, L. Xiao, Y. Ao, J. Zhang and M. Li, A study on regulating the conjugate position of NLO chromophores for reducing the dipole moment and enhancing the electro-optic activities of organic materials, J. Mater. Chem. C, 2020, 8, 1380-1390.

25 C. Rothe, D. Neusser, N. Hoppe, K. Dirnberger, W. Vogel, S. Gámez-Valenzuela, J. T. L. Navarrete, B. Villacampa, M. Berroth, M. C. R. Delgado and S. Ludwigs, Push-pull thiophene chromophores for electro-optic applications: from 1D linear to $\beta$-branched structures, Phys. Chem. Chem. Phys., 2020, 22, 2283-2294.

26 P. Chen, X. Yin, Y. Xie, S. Li, S. Luo, H. Zeng, G. Guo, Q. Li and Z. Li, FTC-containing molecules: large second-order nonlinear optical performance and excellent thermal stability, and the key development of the "Isolation Chromophore" concept, J. Mater. Chem. C, 2016, 4, 11474-11481.

27 A. Zhang, H. Xiao, S. Cong, M. Zhang, H. Zhang, S. Bo, Q. Wang, Z. Zhen and X. Liu, A systematic study of the structure-property relationship of a series of nonlinear optical (NLO) julolidinyl-based chromophores with a thieno[3,2-b]thiophene moiety, J. Mater. Chem. C, 2015, 3, 370-381.

28 Y. Yang, F. Liu, H. Wang, M. Zhang, H. Xu, S. Bo, J. Liu, L. Qiu, Z. Zhen and X. Liu, Synthesis and characterization of a novel second-order nonlinear optical chromophore based on a new julolidine donor, Phys. Chem. Chem. Phys., 2014, 16, 20209-20215.

29 B. Carlotti, A. Cesaretti, O. Cannelli, T. Giovannini, C. Cappelli, C. Bonaccorso, C. G. Fortuna, F. Elisei and A. Spalletti, Evaluation of hyperpolarizability from the solvatochromic method: Thiophene containing push-pull cationic dyes as a case study, J. Phys. Chem. C, 2018, 122, 2285-2296.

30 B. H. Robinson, L. R. Dalton, A. W. Harper, A. Ren, F. Wang, C. Zhang, G. Todorova, M. Lee, R. Aniszfeld, S. Garner, A. Chen, W. H. Steier, S. Houbrechte, A. Persoons, I. Ledoux, J. Zyss and A. K. Y. Jen, The molecular and supramolecular engineering of polymeric electro-optic materials, Chem. Phys., 1999, 245, 35-50.

31 G. Xu, Z. Liu, J. Ma, B. Liu, S. T. Ho, L. Wang, P. Zhu, T. J. Marks, J. Luo and A. K. Y. Jen, Organic electro-optic modulator using transparent conducting oxides as electrodes, Opt. Express, 2005, 13, 7380-7385.

32 C. T. DeRose, Y. Enami, C. Loychik, R. A. Norwood, D. Mathine, M. Fallahi, N. Peyghambarian, J. D. Luo, A. K. Y. Jen, M. Kathaperumal and M. Yamamoto, Pockel's coefficient enhancement of poled electro-optic polymers with a hybrid organic-inorganic sol-gel cladding layer, Appl. Phys. Lett., 2006, 89, 131102. 
33 B. A. Block, T. R. Younkin, P. S. Davids, M. R. Reshotko, P. Chang, B. M. Polishak, S. Huang, J. Luo and A. K. Y. Jen, Electro-optic polymer cladding ring resonator modulators, Opt. Express, 2008, 16, 18326-18333.

34 G. Niu, X. Zheng, Z. Zhao, H. Zhang, J. Wang, X. He, Y. Chen, X. Shi, C. Ma, R. T. K. Kwok, J. W. Y. Lam, H. H. Y. Sung, I. D. Williams, K. S. Wong, P. Wang and B. Z. Tang, Functionalized Acrylonitriles with AggregationInduced Emission: Structure Tuning by Simple ReactionCondition Variation, Efficient Red Emission, and Two-Photon Bioimaging, J. Am. Chem. Soc., 2019, 141, 15111-15120.

35 Z. Shi, J. Luo, S. Huang, X. H. Zhou, T. D. Kim, Y. J. Cheng, B. M. Polishak, T. R. Younkin, B. A. Block and A. K. Y. Jen, Reinforced site isolation leading to remarkable thermal stability and high electrooptic activities in cross-linked nonlinear optical dendrimers, Chem. Mater., 2008, 20, 6372-6377.

36 W. Wang, J. Wu, K. Chen, Q. Huang, J. Luo and K. S. Chiang, Graphene electrodes for electric poling of electro-optic polymer films, Opt. Lett., 2020, 45, 2383-2386.

37 D. Zhang, J. Zou, W. Wang, Q. Yu, G. Deng, J. Wu, Z. A. Li and J. Luo, Systematic study of the structure-property relationship of a series of near-infrared absorbing push-pull heptamethine chromophores for electro-optics, Sci. China: Chem., 2021, 64, 263-273.

38 R. H. Page, M. C. Jurich, B. Reck, A. Sen, R. J. Twieg, J. D. Swalen, G. C. Bjorklund and C. G. Willson, Electrochromic and optical waveguide studies of corona-poled electro-optic polymer films, J. Opt. Soc. Am. B, 1990, 7, 1239-1250.

39 M. G. Kuzyk and C. W. Dirk, Characterization Techniques and Tabulations for Organic Nonlinear Optical Materials, Marcel Dekker, 1998.

40 K. D. Singer, M. G. Kuzyk and J. E. Sohn, Second-order nonlinear-optical processes in orientationally ordered materials: relationship between molecular and macroscopic properties, J. Opt. Soc. Am. B, 1987, 4, 968-976.

41 D. M. Burland, R. D. Miller and C. A. Walsh, Second-order nonlinearity in poled-polymer systems, Chem. Rev., 1994, 94, 31-75.

42 M. Stähelin, B. Zysset, M. Ahlheim, S. R. Marder, P. V. Bedworth, C. Runser, M. Barzoukas and A. Fort, Nonlinear optical properties of push-pull polyenes for electro-optics, J. Opt. Soc. Am. B, 1996, 13, 2401-2407.

43 S. Liu, M. A. Haller, H. Ma, L. R. Dalton, S. H. Jang and A. K. Y. Jen, Focused Microwave-Assisted Synthesis of 2,5Dihydrofuran Derivatives as Electron Acceptors for Highly Efficient Nonlinear Optical Chromophores, Adv. Mater., 2003, 15, 603-607.

44 S.-H. Jang, J. Luo, N. M. Tucker, A. Leclercq, E. Zojer, M. A. Haller, T.-D. Kim, J.-W. Kang, K. Firestone, D. Bale, D. Lao, J. B. Benedict, D. Cohen, W. Kaminsky, B. Kahr, J.-L. Brédas, P. Reid, L. R. Dalton and A. K. Y. Jen, Pyrroline chromophores for electro-optics, Chem. Mater., 2006, 18, 2982-2988.

45 T. L. Kinnibrugh, T. V. Timofeeva, O. Clot, A. Akelaitis and L. R. Dalton, A non-linear optical chromophore: 2-[3-cyano-4((E)-\{5-[4-(diethylamino)styryl]thiophen-2-yl\}vinyl)-5,5-dimethylfuran-2-(5H)-ylidene]propanedinitrile, Acta Crystallogr., Sect. E: Struct. Rep. Online, 2006, 62, o4804-04806.

46 J. Luo, X. H. Zhou and A. K. Y. Jen, Rational molecular design and supramolecular assembly of highly efficient organic electro-optic materials, J. Mater. Chem., 2009, 19, 7410-7424.

47 S. Li, M. Li, J. Qin, M. Tong, X. Chen, T. Liu, Y. Fu, S. Wu and Z. Su, Synthesis, crystal structures and nonlinear optical properties of three TCF-based chromophores, CrystEngComm, 2009, 11, 589-596. 